\title{
Organising a counselling service for problems related to the acquired immune deficiency syndrome (AIDS)
}

\author{
DAVID MILLER,* JOHN GREEN,* AND ALANA McCREANER $\dagger$ \\ From the Departments of *Clinical Psychology and †Genitourinary Medicine, St Mary's Hospital, London
}

SUMMARY Recent Department of Health and Social Security (DHSS) guidelines on screening procedures for antibody to human T cell lymphotropic virus type III (HTLV-III) have stressed the importance of providing adequate counselling for patients before and after testing. The establishment of a national acquired immune deficiency syndrome (AIDS) counselling training unit has enabled large numbers of National Health Service staff to be trained in this work. Suggestions for the establishment of district counselling facilities are made, based on this training, and some preliminary results showing change from high risk sexual behaviour after counselling are presented.

\section{Introduction}

With the advent of publicly available screening for antibodies to human T cell lymphotropic virus type III (HTLV-III) by blood transfusion services, genitourinary medicine departments, and general practitioners, the need for counselling for people seeking such screening and those found to be seropositive is now of paramount importance. Patients contemplating seeking such screening must be alerted to the implications of being found seropositive, and those with positive test results must be made aware of the meaning of this result. Furthermore, those who are asymptomatic, those who develop symptoms of HTLV-III infection, and those who go on to develop AIDS usually require counselling as part of the process of adjusting to the condition.

\section{Groups of patients}

In practice, five groups of patients have emerged:

(1) Those who request screening for antibodies to HTLV-III because of anxieties over possible infection ("worried well" patients). This group includes members of identified high risk groups (homosexual men, bisexual men, haemophiliacs, intravenous drug abusers, and possibly prostitutes). It also includes people from low risk groups who fear they may have

Address for reprints: Mr D Miller, Department of Clinical Psychology, Paterson Wing. St Mary's Hospital, Praed Street, London W2 1NY

Accepted for publication 12 December 1985 become infected with HTLV-III through (often covert) sexual activities, such as promiscuous and non-monogamous married heterosexual men and women and homosexual men with low level of sexual activity. People who develop anxiety after surgery and other invasive medical procedures associated with donated blood and blood products are also classified in this group.

(2) People who are HTLV-III seropositive at screening but have no symptoms of infection. These are usually homosexual men, haemophiliacs, or intravenous drug users, but also include women planning pregnancies or those diagnosed as seropositive during pregnancy:

(3) People who are seropositive for HTLV-III and have symptoms, particularly persistent generalised lymphadenopathy, and who have a sporadic pattern of fatigue, general malaise, and night sweats but do not have AIDS.

(4) People with AIDS and AIDS related complex. The latter patients are highly symptomatic of HTLVIII infection, have evidence of depressed immune functioning, but do not fulfill the current Centers for Disease Control criteria for AIDS. ${ }^{1}$

(5) The sexual partners (lovers), close family members, and friends of those who have been diagnosed as being in the above categories.

Table I shows the proportion of patients in each category from 200 consecutive people seen for counselling at this hospital before the introduction of mass screening facilities in October 1985. 
TABLE I Categories of 200 patients counselled about the acquired immune deficiency syndrome (AIDS)

\begin{tabular}{lr}
\hline Category of patient & $N o(\%)$ \\
\hline Requesting screening ("worried well") & $36(18)$ \\
HTLV-III seropositive but asymptomatic & $34(17)$ \\
HTLV-III seropositive with persistent & $51(25)$ \\
generalised lymphadenopathy & $70(35)$ \\
With AIDS or AIDS related complex & $9(5)$ \\
Lovers, members of families, and friends & \\
\hline
\end{tabular}

* Each patient was a primary referral to counselling, almost all of which resulted in other people being counse!led.

\section{Essential counselling information for the various groups} of patients

THOSE REQUESTING COUNSELLING ("WORRIED WELL" PATIENTS)

For those who come into a screening unit asking to have their blood tested for antibodies to HTLV-III, counselling before testing is essential. Though a screened patient's HTLV-III antibody status is confidential and cannot be disclosed to outsiders without the patient's consent, ${ }^{2}$ knowledge of HTLV-III seropositivity currently prevents patients obtaining any form of life insurance (thus, for example, making it impossible for them to take out mortgages for homes). Furthermore, limited public understanding of the meaning of being seropositive may, if a person's status becomes known to employers and friends, result in loss of work or dismissal and possible social isolation, together with considerable personal suffering. ${ }^{3}$ For instance, patients may be unable to obtain new employment because of the requirement of many companies for health questionnaires and medical examinations.

Many groups of patients in England and abroad have argued that knowledge of seropositivity is meaningless in a medical sense anyway, as it currently provides no predictive accuracy in assessing who of those diagnosed seropositive will eventually develop AIDS. Patients are left, they argue, with distressing information that cannot be supplemented with any firm assurances for the future. Furthermore, whether patients are seropositive or seronegative, the information given concerning risk reduction is identical. For these reasons, counselling before screening must be viewed as a necessary measure. ${ }^{45}$ Patients must be able to make informed decisions about whether to be screened.

THOSE WHO ARE HTLV-III SEROPOSITIVE BUT ASYMPTOMATIC

Patients who have already received a positive HTLVIII result, require counselling to enable them to come to terms with the infection and, just as important, to educate them about ways of preventing the spread of their infection. HTLV-III is a sexually transmissible virus and, in addition to the risk of infecting others by high risk sexual behaviour, those who are infected with the virus are probably more likely to develop AIDS if they also acquire sexually transmitted diseases (STDs). Education on safer sexual behaviour to prevent mutual infection of sexual partners therefore forms an important part of counselling. Similarly, pregnancy in women with HTLV-III infection seems to pose a grave risk to the mother and the foetus, ${ }^{5}$ so it is essential that young seropositive women be counselled about contraception.

Other potential ways of transmitting infection (such as in the home, recreationally, or at work) are very limited, and this fact must be clearly explained to patients so that they can pass this information on to chosen other people equally clearly. ${ }^{6}$ In addition, patients will usually seek advice on how best to minimise the prospect of future health decline. Advice on appropriate diet, recreational drink and drug use, sleep, and exercise regimens may be provided, together with the suggestion of regular six monthly health monitoring at their STD clinic. Further psychological issues associated with a positive HTLVIII result are discussed below.

THOSE WHO ARE SEROPOSITIVE AND SYMPTOMATIC In addition to the risk reduction advice concerning safer sex, infection control, and "health boosting" that asymptomatic seropositive patients should receive routinely, symptomatic patients have a higher risk of developing AIDS, which creates enormous psychological burdens that can be reduced by informed counselling. ${ }^{3}$ These burdens are increased when patients are required to cope with the often incapacitating physical symptoms that accompany the condition. The impact of symptoms on their ability to work and socialise commonly results in withdrawal and depression, which is often compounded by the restrictions on their previous sexual behaviour and the cloak of secrecy drawn over their diagnosis. The increasing rate of suicide attempts in this population, combined with the possibility that chronic anxiety and depression may in themselves be immunosuppresive, ${ }^{7}$ points to the need for early and effective counselling.

PATIENTS WITH AIDS AND AIDS RELATED COMPLEX In counselling people in this group, the essential information mentioned above is equally relevant and should be presented to them. In addition, however, people with AIDS have to face the possibility of their own death more directly. This is not to say that they will necessarily be under greater psychological pressure than those in other groups of patients. Many respond to a diagnosis of AIDS with a sense of relief, for they may have suspected for some time that they have AIDS and a firm diagnosis at least relieves this uncertainty. For many, however, the emotional impact of the diagnosis is enormous, particularly in 
younger patients who have never been seriously ill before. The range of issues that must be confronted in the process of long term adjustment is very wide, ${ }^{8}$ and the necessity for a co-ordinated counselling structure giving links with psychiatry, psychology, and relevant medical, community, and religious authorities to meet the needs highlighted in this context is obvious.

\section{LOVERS, FAMILIES, AND FRIENDS OF PATIENTS}

Few seropositive patients are entirely without important social links. Many have lovers and, because safer sexual activity to prevent transmission of the virus and the acquisition of other infections are usually more restrictive than previous sexual activities, their sexual partners need to be informed. They will then often respond with anxieties about their own antibody status as well as fears about the wellbeing of their lovers or other sexual partners. The risks posed by living, working, and socialising with an HTLV-III seropositive person are not well understood by the public at large (as recent media coverage of schoolchildren with haemophilia has shown), and clearly people living with, and sharing the frustrations of, those infected need to be supported and educated as fully as possible. Experience with the lovers of seropositive patients suggests that the rates of psychological morbidity are in many cases higher in them than in the patients. This is probably for two reasons: (1) because they are "sharing" the diagnosis in a functional sense - that is, they become as restricted socially and sexually as the patient and have to "nurse" them continually through the process of adjustment to the diagnosis - and (2) they may often have worries about their own antibody status if, for instance, they have been having high risk sexual intercourse with the patient for many months or years.

\section{Organising a counselling service}

SITING

Untıl recently, clinics of genitourinary medicine (GUM) or sexually transmitted disease (STD) were the main centres for screening, and it was such clinics that the group at highest risk for HTLV-III, homosexual men, conventionally attended. Similarly, haemophilia resource centres provided counselling for haemophiliacs and their sexual partners who had been infected with HTLV-III through contaminated factor VIII and factor IX. Now that all blood donated to the blood transfusion service is screened for antibody to HTLV-III, and general practitioners can request screening, it is clear that both the blood transfusion service and general practitioners must have access to trained counsellors, or be prepared to provide such counselling themselves.

Not all patients are likely to be seen as outpatients. Some will be identified when they are admitted to hospital for treatment for AIDS and AIDS related problems. Staff caring for large numbers of such patients should clearly have, or have access to, the appropriate counselling skills. As the incidence of HTLV-III infection rises in the United Kingdom virtually every hospital department will probably eventually come into contact with seropositive patients.

\section{COUNSELLING STAFF}

Experience has shown that particular skills are required of counsellors for people with AIDS related problems. They must have basic counselling skills. This does not necessarily mean counselling qualifications; natural abilities are often more important and useful than formal qualifications. They must be well informed about AIDS, HTLV-III, and relevant symptoms, as well as lifestyles associated with high risk groups. They must also be able to keep up with developments in a topic in which medical and social understanding are changing rapidly. They must be good communicators, and be able to deal with a wide range of hospital staff effectively and tactfully. They should be well informed about the range of "back up" staff in their district and community. They must be able to recognise the common clinical complications related to AIDS, such as anxiety, depression, obsessive or compulsive behaviour, neurological disorders, and the risk of suicide. They must be able to treat such complications themselves, or to recognise when to refer patients elsewhere.

The possession of the relevant information, skills, and interest is more important than the profession from which the counsellor comes. In the context of GUM, blood donor, and haemophilia clinics the obvious choice is the doctor who gives the patient the test results. The counsellor must recognise, however, that intervention is rarely a simple "one off" chat, and if the patient's doctor does not have the time to provide follow up counselling, it is clearly advisable that another professional, perhaps a health adviser or clinic nurse, carries out the counselling. Particularly in the context of GUM or STD clinics, such staff will already possess valuable experience in dealing with STDs and managing the social problems they cause.

Some health districts may have available psychologists or psychiatrists with an interest in this work. Such staff have the advantage of extensive experience of managing patients in distress. It is important to establish links with psychiatric services as many patients do develop frank psychiatric states requiring admission to hospital, such as suicidal depression.

With inpatients the most likely counsellors will be the nursing staff. They have the most extended daily contact with patients, and it is to them that patients will often present their concerns. They are also likely 
to be the main points of contact between relatives, partners, and friends of the patients and the hospital.

\section{STRUCTURE OF COUNSELLING SERVICE}

Whether or not the counsellor does only work related to AIDS, and the number of staff required for such work, depends very much on the local circumstances and the number of patients being seen. For example, blood transfusion service facilities may cover a large geographical area and, instead of a member of staff spending a long time travelling hundreds of miles to see widely spaced patients, a more cost effective solution would be to identify a network of local resource staff to follow up local needs. This clearly requires co-ordination throughout and between districts in the early stages, but could save much time and individual suffering in the long term. The national AIDS counselling training unit that has been established at this hospital can provide continuing training of strategically placed staff to ensure that quality control in counselling is maintained, and can ensure cost effective links between key counselling staff.

The structure of a counselling service can best be shown by considering patients coming for screening for HTLV-III. The figure shows the various stages through which they may go.

The first stage of intervention is at the time the HTLV-III antibody test is requested. The first question is whether or not the test should be undertaken. This can be decided usually by discussing with the patient the implications of a positive result in medical, emotional, sexual, employment, and financial terms. It should be stressed that behavioural recommendations are the same whether the results are positive or negative. It is interesting that in our experience the most appreciable and sustained changes in sexual behaviour come when a positive diagnosis is confirmed, which suggests that viral infection is the most profound motivator (see below). It is also interesting that in many cases the financial (insurance and mortgage) consequences of counselling before testing are the deciding factors against being tested.

Counselling before testing may appear to be a doctrine of perfection. In many busy and understaffed clinics, the practical difficulties in allocating precious staff time to large numbers of patients will initially outweigh the otherwise clear responsibility to provide adequate information and consideration before screening. Many clinics see patients more or less on demand, and the reasons for their attending will not be known in advance. On the other hand, the implications of being tested are enormous for the patient. Many people coming for screening will probably have to be given fixed appointments at a later date so that counselling before testing can be achieved.

If, after counselling, the person decides to go ahead with testing and a blood sample is taken, the second stage necessitates the patient returning to the place of screening for the results of the test. It should be possible to arrange an appointment system for this, so that a counsellor can be on hand. Again, this should be so whether the patient is seropositive or seronegative,

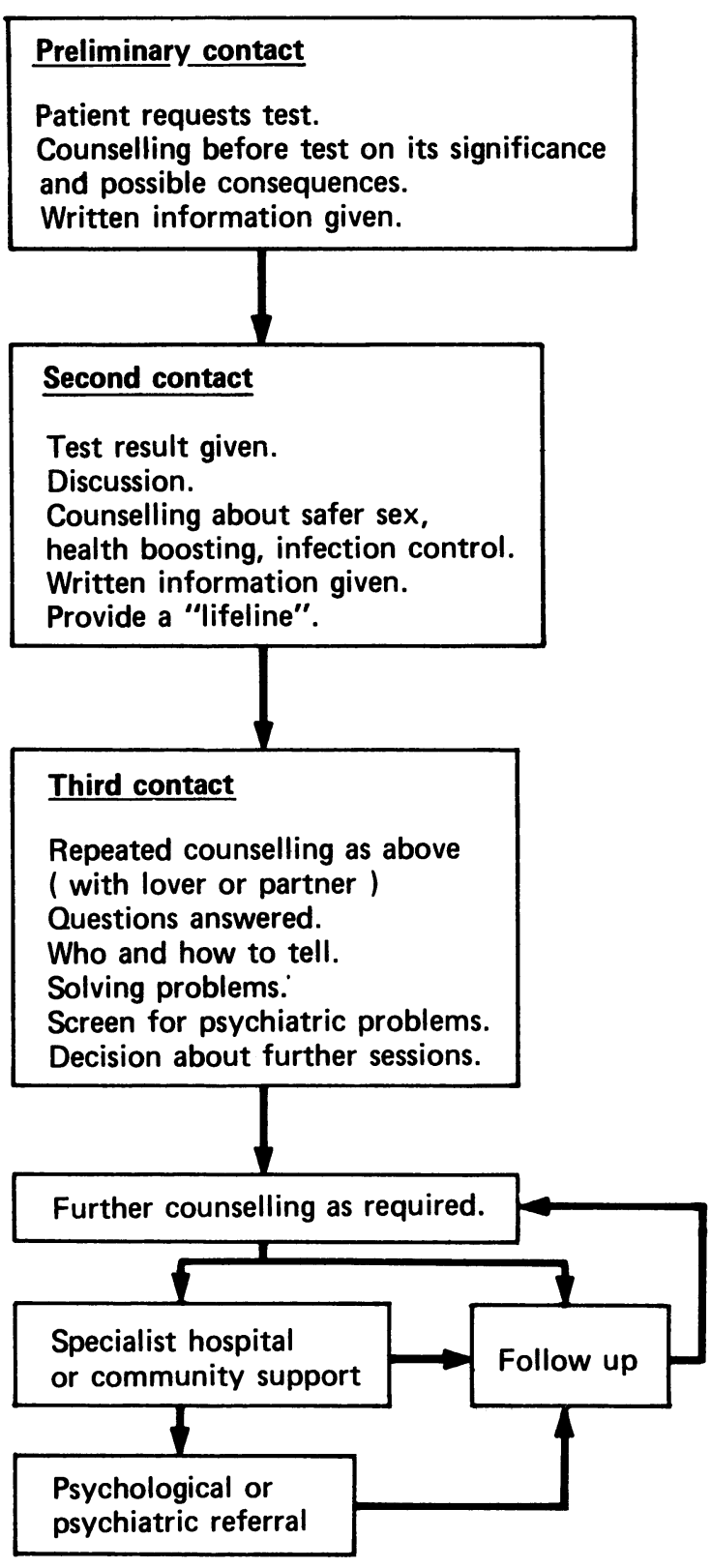

FIGURE Stages of counselling for people requesting tests for antibodies to human $T$ cell lymphotropic virus (HTLV-III). 
as the same advice on infection control will be required for the patient's future activity. One problem is that patients are often very anxious when they come in for their results, and high levels of shock and anxiety accompany positive results. ${ }^{4}$ Similarly, a negative result will often produce euphoria. In either case information received is likely to be distorted and misunderstood, so it is advisable to allow plenty of time for the result to be accepted and then to repeat the essential information, perhaps supplementing discussion with written summaries.

In subsequent sessions, as anxiety reduces and the result is absorbed by the patient, information may need to be repeated. It is also necessary to include in the discussions topics such as "who and how to tell" and "solving problems", and to assess the patient for signs of psychological or psychiatric disturbance. If the patient appears to be managing successfully, the decision to end current intervention can be taken, typically at the third session after testing. The appearance of residual adjustment difficulties, including those arising in the context of a stable relationship, may indicate the need for further sessions, or perhaps referral to other psychiatric or community support specialist agencies.

It is useful and practical to give outpatients a telephone number (a "lifeline") which they can use to ask questions or make further appointments. Many patients find such a resource particularly reassuring and, rather than creating a flood of time consuming calls from distressed people, such a provision seems to be both time saving and successful in maintaining patient compliance. Queries are typically straightforward, and being able to ask them by telephone saves the patient much unnecessary time and anxiety waiting for appointments.

Symptomatic patients, such as those with persistent generalised lymphadenopathy, AIDS related complex, and AIDS, are likely to need longer term support than other diagnostic groups. For the inpatient with AIDS it is important that a counsellor be available as soon as possible after diagnosis, and that consistent and regular contact be provided.

\section{GENERAL CONSIDERATIONS}

Several considerations apply to AIDS related counselling. The first is that a wide range of staff will see patients, particularly those with persistent generalised lymphadenopathy, AIDS related complex, and AIDS. It is vital that they should all give the same information, and interpretations of that information, to the patient. Differing information from health professionals leads to confusion, suspicion, and reduced compliance by the patient. Thus a clear institutional policy on patient management, and clear mechanisms for staff liaison and discussion, are necessary. A regular "outpatient round" for all staff concerned is often helpful for them to get up to date on the medical and social management of the patients.

A second consideration is that most people concerned with particular patients will, to some extent, be counselling them. It is therefore important that one person should take overall responsibility for co-ordinating the counselling input and that that person's role should be recognised by other staff concerned. Consistency is vital, as confusion will result if everyone is using their pet approach with the patient.

Thirdly, working with people with AIDS and AIDS related problems can be very distressing, and it is often helpful to provide staff with a "support group" in which their anxieties, frustrations, and emotions can be constructively ventilated. People are usually relieved to find that "specialists" have the same difficulties as they do, specially when patients are slowly dying, and their lovers and relatives are crying and sometimes aggressive. Such discussions often produce useful ideas that can improve the system for all.

Finally, if a unit has large numbers of patients, setting up a self help or support group for patients may be worth considering. It is better generally to have specific groups for specific diagnostic categories, and the counsellor may help to organise and facilitate these meetings. Such groups appear to have considerable benefits for newly diagnosed patients, as they can benefit from the experiences of "veteran" members.

\section{Preliminary results of counselling on high risk behaviour}

From 112 consecutive patients studied at this hospital between February and June 1985, including many who were seropositive but asymptomatic, seronegative, or sexual partners of identified seropositive patients, some indication of the impact of counselling on high risk sexual behaviour can be made. Each patient was given counselling on safer sexual procedures; on domestic, occupational, social, and recreational infection control; on risk reduction; and on "health boosting". Furthermore, hospital based counselling was supplemented with the offer of joining community based support organisations (such as the London based self help group "Body Positive"), which could maintain patient counselling and motivation. Three months after the initial period of counselling, patients were each asked about the type

TABLE II Numbers in various types of relationship before and after counselling of 112 patients.

\begin{tabular}{lll}
\hline Relationship & Before & After \\
\hline Monogamous relationship & 16 & 37 \\
Open relationship & 39 & 6 \\
No stable relationship & 57 & 69 \\
\hline
\end{tabular}


TABLE III Effects of counselling on sexual behaviour within couples and relationships

\begin{tabular}{lcc}
\hline Sexual behaviour & Before & After \\
\hline Monogamous couples: & & \\
No sexual activity & 0 & 1 \\
Safe sexual activity only & 0 & 20 \\
Anal intercourse with condom & 12 & 2 \\
Anal intercourse without condom & & \\
& & \\
Open relationships: & 0 & 0 \\
No sexual activity & 9 & 4 \\
Safe sexual activity & 0 & 2 \\
Anal intercourse with condom & 30 & 0 \\
Anal intercourse without condom & & \\
& & \\
No stable relationship: & 2 & 17 \\
No sexual activity & 4 & 45 \\
Safe sexual activity & 0 & 4 \\
Anal intercourse with condom & 51 & 3 \\
Anal intercourse without condom & & \\
\hline
\end{tabular}

and extent of safer sexual activity they had maintained. Tables II and III show the results.

These figures show that counselling has motivated a substantial move away from non-monogamous stable relationships to either monogamy or no stable relationship in which celibacy or safe sexual activity predominates. Within monogamous relationships the interesting feature was the large number of couples who maintained anal intercourse with condoms and lubricants. As unprotected anal intercourse had generally been practiced before counselling, this was a major change (despite the unproved protection by condoms from transmission of HTLV-III). Also appreciable was the number (14) of monogamous couples who changed to safe sexual activity with not even protected anal intercourse.

The figures for changes in sexual behaviour in open stable relationships indicated similar trends towards attempts to reduce viral transmission. Similarly, counselling appeared to have resulted in greater numbers of people practicing safe sexual activity or celibacy.

The apparent "polarising" effect of counselling was interesting. Within many stable relationships, one partner remains monogamous or celibate, whereas the other has a number of "external" sexual partners. Counselling seemed to result in either a "bonding", which incorporated an increase of safe sex for the people concerned, or a "splitting" and separation, which resulted in an increase in safe sex or maintained celibacy. Of particular interest is the return to monogamy of the married bisexual men in this sample (4/112), two of whom were seropositive and adopted the use of condoms.

The number of people who did not modify their sexual lifestyle and who continued with unprotected anal intercourse seems reassuringly low (5/112) considering the multiplicity of values within the many sections of the homosexual community interviewed.

Some further issues were hightlighted by this survey. After being informed that they were seropositive, many men experienced a complete loss of sexual drive. Experience with longer term patients suggests this is often only a temporary effect. Sexual activity with others was often replaced by masturbation associated with videos, magazines, and books. Many people reported feeling they had changed their sexual behaviour for ever, and most reported that the "gay scene" would never be the same. Importantly, all those making sexual behavioural changes reported a reduction in the numbers of sexual contacts (to an average of three) during the three month follow up. The peer group influence of community based support groups was often held to be important in motivating this reduction, where it occurred, and in maintaining compliance with safe sex advice.

The group studied was unusual in a number of respects. Most patients in the group were seropositive and, because they were seen before the availability of testing on request, most were either symptomatic or knew themselves to be the sexual contacts of people with AIDS. The group contained a high number of patients with frank AIDS. These factors may have increased the success rate of counselling. None the less, the results obtained are extremely promising and it is now important to measure the success of counselling in patients attending for screening on request. Further information is also needed on the effects of counselling on those who do not wish to be tested.

The follow up period was also short, and changes in sexual behaviour should be followed up for a longer period to assess how long changes are maintained beyond three months.

\section{Conclusion}

Clearly this limited follow up survey indicates that the establishment of a well organised counselling structure leads to effective changes in sexual behaviour to limit future virus transmission. Data need to be obtained from other groups of patients, including those who do not wish to be tested and those attending for testing on request. The importance of peer group pressure and other influences, such as the variety and intensity of counselling, must be analysed over longer periods before such intervention can be regarded as more than just very promising in its effects. Research into these important issues is currently being undertaken.

\section{References}

1. Weber J, Pinching AJ. Clinical management of AIDS and HTLV-III infection. In: Miller D, Weber J, Green J, eds. The management of AIDS patients. London: The Macmillan Press, 1986:1-33. 
2. Department of Health and Social Security. AIDS Booklet 2. information for doctors concerning the introduction of the HTLVIII antibody test. Heywood, Lancashire: DHSS Health Publications Unit, October 1985.

3. Miller D, Green J. Psychological support and counselling for patients with acquired immune deficiency syndrome (AIDS). Genitourin Med 1985;61:273-8.

4. Miller D, Weber J, Green J, eds. The management of AIDS patients. London: The Macmillan Press, 1986.

5. Pinching AJ, Jeffries DJ. AIDS and HTLV-III infection: consequences for obstetrics and perinatal medicine. J Obstel Gynaecol (in press).
6. Green J. Counselling HTLV-III seropositives. In: Miller D, Weber J, Green J, eds. The management of AIDS patients. London: The Macmillan Press, 1986:151-68.

7. Anonymous. Emotion and immunity. Lancet 1985; ii:133-4.

8. Miller D. Psychology, AIDS, ARC and PGL. In: Miller D, Weber J, Green J, eds. The management of AIDS patients. London: The Macmillan Press, 1986:131-49. 